Proc. 13th International School on Theoretical Physics: Symmetry and Structural Properties of Condensed Matter

\title{
The Application of the Special Functions to Solving Physical Problems
}

\author{
A. WŁOCH ${ }^{a *}, \mathrm{H} . \mathrm{CZYZ}^{b}$ AND T. JASIŃSKI ${ }^{b}$ \\ ${ }^{a}$ Department of Mathematical Modeling, Faculty of Mathematics and Applied Physics, \\ Rzeszów University of Technology, al. Powstańców Warszawy 12, 35-959 Rzeszów, Poland \\ ${ }^{b}$ Department of Physics and Medical Engineering, Faculty of Mathematics and Applied Physics, \\ Rzeszów University of Technology, al. Powstańców Warszawy 12, 35-959 Rzeszów, Poland
}

\begin{abstract}
The purpose of this work is to examine the reflection of a plane acoustic wave at normal incidence at the boundary of equally flat medium of finite thickness. It separates two homogeneous semi-infinite media. We are interested in the case where two external media have constant wave numbers, respectively $k_{1}$ and $k_{2}$, and that the wave number $k$ varies from $k_{1}$ to $k_{2}$ in a continuous and monotonic way. This is a problem that we solve by using Mathieu's functions.
\end{abstract}

DOI: 10.12693/APhysPolA.135.1234

PACS/topics: special functions, Mathieu functions, mechanical waves propagation

\section{Introduction}

The aim of this paper is to present examples of the new application of the special functions to solving problems in the field of theoretical physics. In the process of theoretical description of a physical phenomenon scientists apply mathematical methods, which may facilitate physical interpretation of the results obtained. Special functions used in the theoretical physics were introduced as a result of the search for the solutions of practical problems. The special functions, among other things Mathieu functions, have application to aspects of mechanical waves related to cylinders, ellipses, and elliptical cylinders, etc. These functions occur during the separation of the variables in a wave equation (with partial derivatives) in various co-ordinate systems. Propagation of a mechanical wave in a liquid having variable wave number $k$ is found in practice quite frequently. This phenomenon is described by a differential equation of variable coefficients i.e. the Helmholtz equation with a variable wave number. This equation may be solved using various methods. Solving equations of this type often poses significant difficulties. The foregoing considerations are different from the ones presented in the literature. Our suggestion is the following: also, a variable wave number, i.e. a $k(x)$ function may be applied, which - having satisfied the assumptions - may result in a differential equation with a known solution, i.e. the Mathieu equation.

\section{Propagation of acoustic wave} in the acoustically non-homogeneous medium

Macroscopic non-homogeneity of a medium caused by a difference in temperature may be expressed in the form

*corresponding author; e-mail: awloch@prz.edu.pl of the wave equation. This equation has one parameter characterizing the medium: velocity of wave propagation c. This velocity is a function of temperature, whereas the medium is acoustically heterogeneous. Propagation of an acoustic wave in a liquid medium with a temperature gradient is found in practice quite frequently. This subject was intensely studied in Great Britain, which has always been a maritime power [1]. Acoustic signaling is a significant aspect of navigation, as acoustic signals carry information on the macrostructure of the sea. An acoustically heterogeneous medium may be conventionally divided into layers inside which the temperature is a function of (distance) height. In research of waves spreading over the sea, the medium is assumed to be layered. The phenomenon of acoustic wave propagation in a nonhomogeneous medium is described by a differential equation of variable coefficients. Solving equations of this type often poses significant difficulties.

\section{Wave reflection and transmission in a liquid medium with a temperature gradient}

Acoustic plane wave propagates in a liquid medium having variable wave number $k$ (i.e. in a medium with a temperature gradient) which separates two media of wave numbers: $k_{1}=$ const and $k_{2}=$ const (i.e. of constant temperatures), whereas $k_{1} \neq k_{2}$.

The media are linked with an intermediary layer, with coefficient $k(x)$ changing constantly and monotonically from $k_{1}$ to $k_{2}$. This is an issue that we solve using the Mathieu function. To calculate the reflection and transmission of a wave in the above-described conditions, an equation must be written for acoustic potential $\Phi(x)$ (assuming that it is a one-dimensional problem) and variable wave number $k(x)$, i.e. the Helmholtz equation with a variable wave number. The Helmholtz equation with a variable wave number may be solved using various methods. The problem has been formulated and solved by 
Brekhovskih [2]. Later, Jessel et al. showed that by means of appropriate change of function, Helmholtz's variable-coefficient equation is split up into a pair of equations of the same type, but with constant coefficients in which "virtual sources" appear containing the unknown field [3]. By using Green's function in expressing the solution, these equations can be transformed into an integrodifferential equation for the field and an integral equation for the virtual sources. The latter equation was solved numerically and hence the coefficient of reflection was deduced. Our suggestion is the following [3]: also, a variable wave number, i.e. a $k(x)$ function may be applied, which - having satisfied the aforesaid assumptions may result in a differential equation with a known solution, i.e. the Mathieu equation [4].

\section{Mathematical description of the problem}

In a liquid medium with a temperature gradient from $T_{1}$ to $T_{2}$, a plane sinusoidal wave propagates vertically to the boundary between the media, over distance $d$, with wave numbers, respectively: in medium $E_{1}, E, E_{2}$ :

$E_{1}$, for $x<0 k(x)=k_{1}=$ const,

$E$, for $0<x<d k(x) \neq$ const,

$E_{2}$, for $x>d k(x)=k_{2}=$ const.

For a plane sinusoidal wave spreading vertically to the boundary between the media, its propagation is described using three equations [3]:

- in the medium of a wave number $k_{1}$ :

$$
\varphi^{\prime \prime}(x)+k_{1}^{2} \varphi(x)=0,
$$

- in the medium of a variable wave number $k(x)$ :

$$
\varphi^{\prime \prime}(x)+k^{2}(x) \varphi(x)=0,
$$

$$
\text { where } k^{2}(x)=a-2 q \cos (2 x) \text {, }
$$

- in the medium with a wave number $k_{2}$.

$$
\varphi^{\prime \prime}(x)+k_{2}^{2} \varphi(x)=0 \text {. }
$$

\section{Solution of the problem}

Solutions of equations for $E_{1}$, and $E_{2}$ are known [2]. A solution for $E$ adopts the form of the Mathieu function [4, 5], see Figs. 1 and 2. Constants are determined from the boundary conditions at the boundary between the media, the continuity of acoustic pressure, and the vibration speed for $x=0$ and $x=d$. The boundary conditions are a system of four equations with four unknowns: $R, A, B, T$, including the wave reflection coefficient $R$ (see Fig. 3 and Fig. 4) at the boundary between the media in the situation analyzed [3].

\section{Discussion}

The foregoing considerations are different from the ones presented in the literature $[1,6]$ taking advantage of the same method as the one used here. The above-described method of solving the problem of wave propagation in a non-homogeneous medium, in the conditions described above, makes it possible to present the results in an analytic form using the Mathieu function. The case in question is a heterogeneous medium, of variable wave number $k(x)$, linking two homogeneous media of constant wave numbers $k_{1}$ and $k_{2}$.

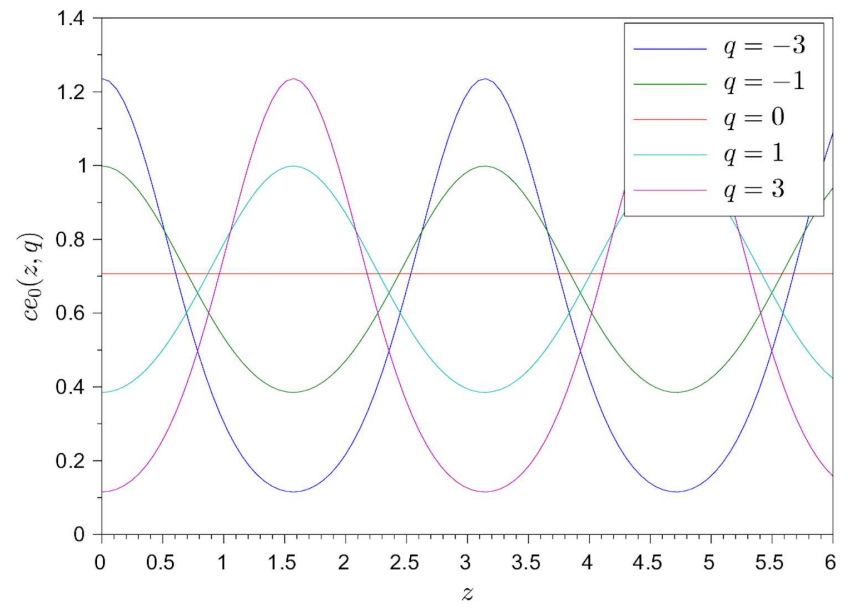

Fig. 1. Even periodic Mathieu functions.

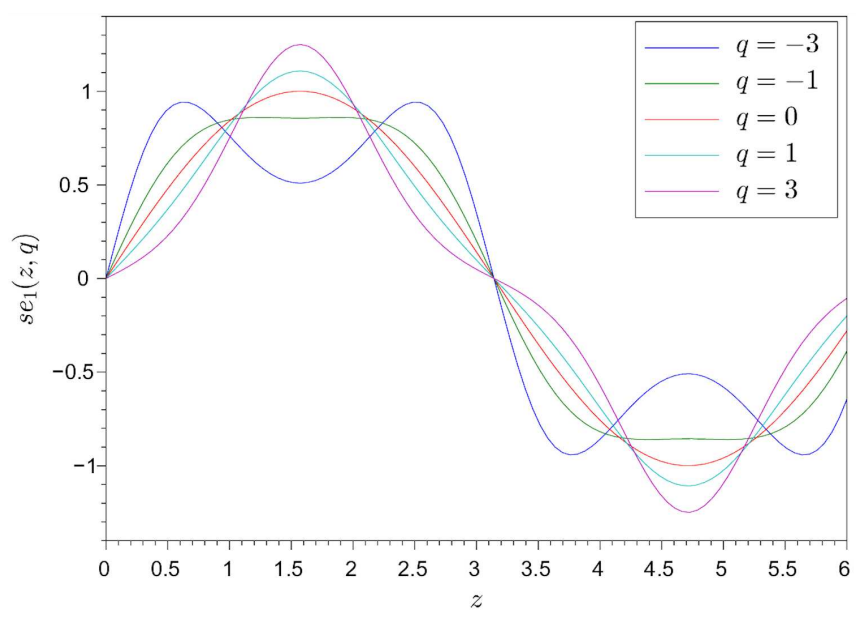

Fig. 2. Odd periodic Mathieu functions.

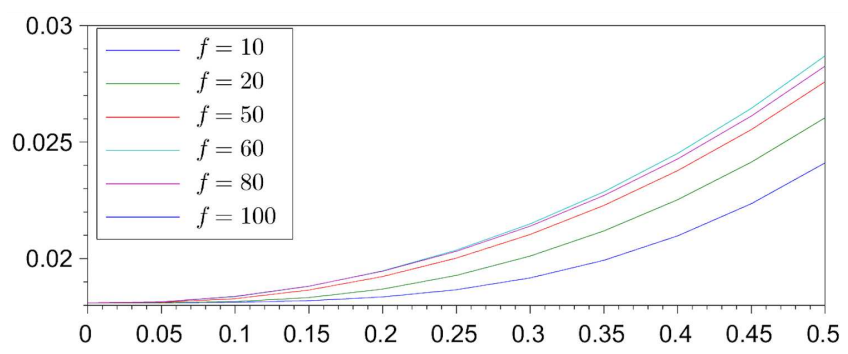

Fig. 3. The coefficient of reflection of the wave $R$ as a function of distance $d<0.5 \mathrm{~m}$ for several values of the wave frequency. 


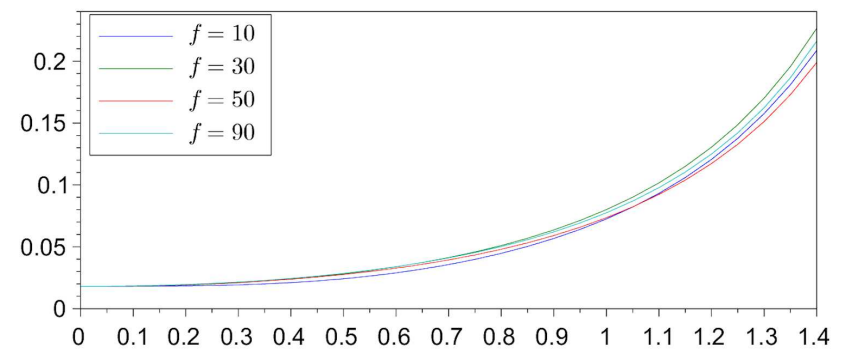

Fig. 4. The coefficient of reflection of the wave $R$ as a function of distance $d<1.4 \mathrm{~m}$ for several values of the wave frequency.

\section{Examples of other applications of the Mathieu function}

- Analysis of the vibrations of water in a lake of an elliptical outline. Assumptions: - movements of water in a lake of constant depth $d$ is stationary all over the plane, - dependence of the vertical movement of water particles $\xi$ on time is $e^{i \omega t}$, - water particle movement $\xi$ is small. The differential equation of movement is in elliptical co-ordinates [4]. The solution to be found is a combination of the Mathieu functions which represent the deformation of water surface. Function $\xi$ represents the configuration of water surface at time $t>0$. The constants occurring in the solution are determined from the initial conditions at $t=0$.

- Analysis of the problem of the plane acoustic wave propagation in a liquid medium in which there is an obstacle - an elliptically shaped cylinder. If there is an obstacle on the way of a spreading acoustic wave, acoustic scattering occurs. It is assumed that the obstacle, e.g. an elliptically-shaped cylinder, is many times longer than the larger axis of the ellipse. The axis of a long elliptical cylinder of an elliptical cross-section is perpendicular to the plane of the page. The medium flows round the elliptical cylinder with velocity $u$ in the direction forming angle $v$ with the larger axis of the cylinder. In this case, solving the diffraction problem requires the use of elliptical co-ordinates, whereas the solution of the wave equation in such co-ordinates includes the Mathieu functions.

\section{Conclusion}

The applications of the Mathieu function to solving acoustic problems may be divided into two main groups:

1. Solutions of the two-dimensional wave equation written using elliptical co-ordinates;

2. Solutions of boundary conditions problems.

The majority of the applications of the Mathieu function falls into the first group of the problems related to the wave equation.

\section{References}

[1] E. Kozaczka, G. Grelowska, Pol. Marit. Res. 24, 48 (2017).

[2] L. George, Am. J. Phys. 67, 165 (1999).

[3] H. Czyż, T. Markowski, Hydroacoustics 9, 23 (2006).

[4] N.W. McLachlan, Theory and Application of Mathieu Functions, Oxford 2015.

[5] R. Coïsson, G. Vernizzi, X. Yang, in: Proc. Conf. Open-Source Software for Scientific Computation (OSSC), IEEE, 2009, IEEE, Guiyang (China) 2010, p. 3.

[6] D.A. Gubaidullin, Yu.V. Fedorov, Acoust. Phys. 64, 164 (2018). 